\title{
Movilidad urbana, transporte público y lineamientos para un plan de movilidad urbana en la Zona Metropolitana de Cancún
}

Urban mobility, public transport and guidelines for an urban mobility plan in the metropolitan area of Cancún

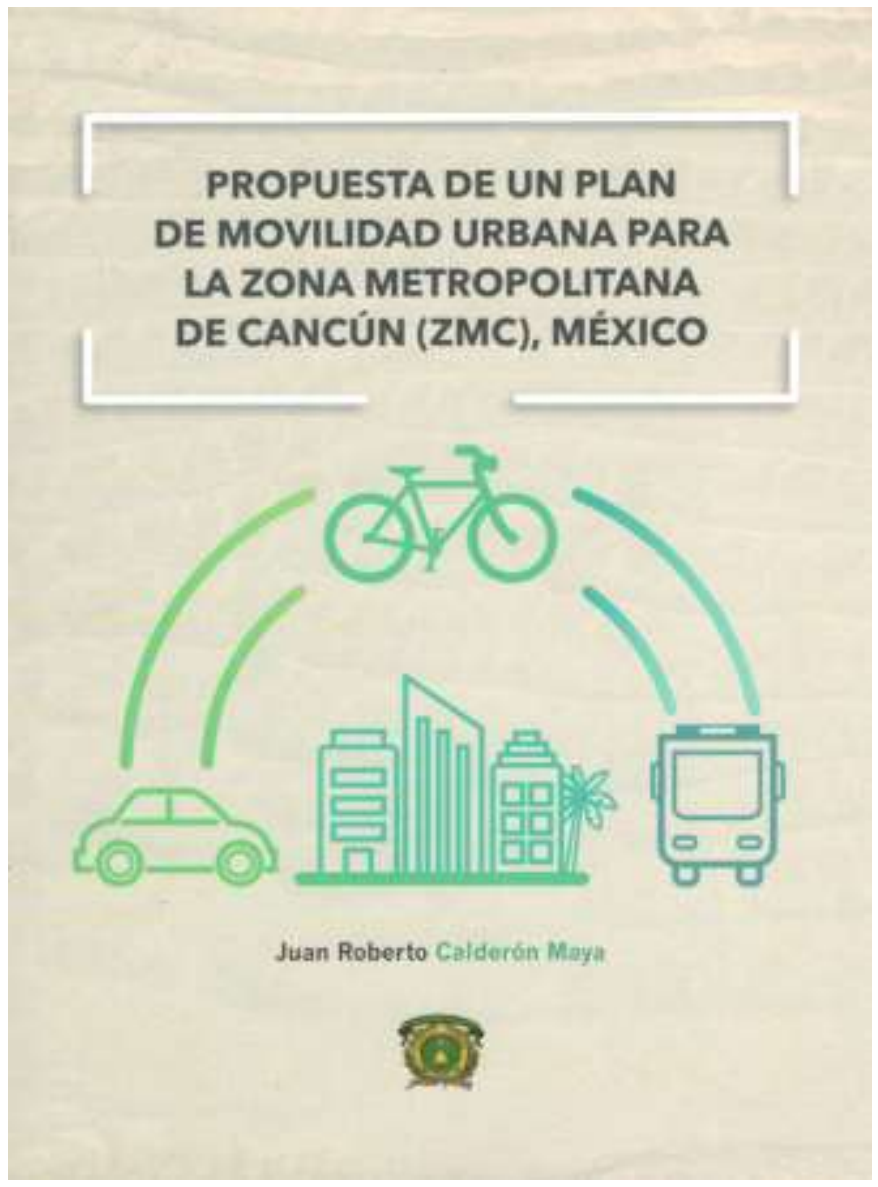

Reseña del libro: Calderón Maya, Juan Roberto. (2019). Propuesta de un plan de movilidad urbana para la Zona Metropolitana de Cancún (ZMC), México. Toluca: Universidad Autónoma del Estado de México, Secretaria de Investigación y Estudios Avanzados y Facultad de Planeación Urbana y Regional.

*Universidad Autónoma del Estado de México, Facultad de Planeación Urbana y Regional, México. Correo electrónico: anjca.du@gmail.com 
Juan Roberto Calderón Maya, autor del libro "Propuesta de un Plan de Movilidad Urbana para la Zona Metropolitana de Cancún (ZMC), México, al que refiere esta reseña, cuenta con experiencia y trayectoria académica fehaciente en la temática que aborda; prueba tangible es la impresión de este texto que, a su vez, es producto de una investigación más amplia de la zona de estudio; de igual manera, ha escrito artículos científicos, otro libro e impartido ponencias sobre el tema.

El autor es Doctor en Urbanismo por la Universidad Autónoma del Estado de México (UAEM); forma parte del Sistema Nacional de Investigadores (SNI) del Consejo Nacional de Ciencia y Tecnología (CONACYT); cuenta con el Reconocimiento Perfil Deseable del Programa para el Desarrollo Profesional Docente (PRODEP); es Director de la Facultad de Planeación Urbana y Regional (FaPUR) en el periodo 2016-2020; ha colaborado como Subdirector Académico y Subdirector Administrativo de la FaPUR. Asimismo, es integrante de la Red de Servicio Voluntario Ambientalista (RESERVA) de la Secretearía del Medio Ambiente del Gobierno del Estado de México desde el año 2007, y colabora en distintas asociaciones entre las que destacan su participación en El Consejo Nacional de Centros Histórico, A.C., la Asociación Nacional de Instituciones de Enseñanza de la Planeación Territorial, el Urbanismo y el Diseño Urbano (ANPUD) y como Representante de la Asociación Latinoamericana de Escuelas y Urbanismo y Planeación (ALEUP).

Calderón tiene poco más de una década realizando trabajo de campo en Cancún colaborando con la Universidad Autónoma de Quintana Roo; en este tiempo ha podido observar, vivir, investigar, experimentar, analizar y reflexionar sobre los problemas existentes en la Zona Metropolitana de Cancún (ZMC), que engloba en tres aspectos relevantes: desarrollo urbano, movilidad y medio ambiente.

Así, la obra se compone de una presentación y justificación, que exponen la perspectiva y el contexto en donde se desarrolló el estudio de la ZMC; con ello, se va orientando al lector en los contenidos que podrá encontrar a lo largo de los cuatro capítulos, los cuales fueron estructurados de manera asertiva y congruente; esto, a su vez, facilita su abordaje individual y apoya a los investigadores a tratar tópicos precisos con la teoría y la conceptualización de la movilidad urbana, el transporte y la metrópoli.

En el capítulo uno, titulado Marco teórico conceptual de la movilidad urbana y el transporte público en la ZMC, el autor logra el propósito de contribuir con un marco teórico de referencia conceptual que permite ubicar al lector en la teoría y en las variables conceptuales. Se divide en tres subcapítulos; el primero esgrime los fundamentos teóricos sobre el fenómeno urbano-metropolitano y la movilidad urbana, y desarrolla el enfoque de las teorías en torno a urbanización, metropolización, estructura urbana, movilidad urbana y transporte; la segunda parte aborda los principales planteamientos relacionados con las variables conceptuales de los enfoques teóricos planteados en el primer subcapítulo, 
urbanización, metropolización, movilidad, transporte, oferta y demanda del transporte público, estructura urbana y accesibilidad al transporte público; en el tercer subcapítulo se muestra el objetivo principal y los aspectos relevantes en los que un plan de movilidad urbana puede contribuir en el desarrollo de un sistema de transporte urbano.

Por tanto, el capítulo uno está bien logrado si consideramos que la intención de Calderón es orientar y ubicar de manera clara y precisa al lector en un punto que permita comprender de manera asertiva las teorías, los conceptos y las variables abordadas en los capítulos subsecuentes, comprendiendo que hay material inicial para investigaciones orientadas, por lo menos, con tres teorías sobre movilidad urbana, transporte público y zonas metropolitanas. Además, la bibliografía utilizada tiene poco más de treinta fuentes y alrededor de setenta y cinco citas, en las cuales retoma a los exponentes más importantes en el tema.

En el capítulo dos, denominado Descripción de casos de éxito de planes de movilidad urbana internacionales, de América Latina y nacionales, Calderón describe el caso internacional de Dresden, Alemania, el cual fue retomado por la reducción del uso del vehículo y por buscar alternativas de desplazamiento en el territorio para una mejor movilidad; otro punto relevante de este plan es su estructura organizacional, ya que involucra a miembros de la sociedad, a políticos de la zona y a los municipios aledaños para conformar un comité encargado de crear el plan de movilidad urbana. Nagpur, India, es otro caso internacional, cuyo plan destaca por la integración del uso del suelo y del transporte, a fin de contar con movilidad segura y sustentable para sus ciudadanos; de este plan, el autor retoma ocho indicadores propuestos por Bohler-Baedeker et al. (2014) para la movilidad urbana, con el propósito de utilizarse en la metodología en el diagnóstico de la ZMC.

Respecto a los casos latinoamericanos, el primero es Bogotá, Colombia; la información abordada es un poco más amplia que en los casos internacionales, como lo advierte el autor en su introducción capitular, pues nos proporciona más datos sobre la situación y el contexto de Bogotá y sobre la relación que guarda el plan de movilidad urbana con otros planes, como el de ordenamiento territorial o las unidades de planeación zonal. De este modo, Calderón rescata cuatro indicadores para su metodología, los cuales le servirán para medir los impactos de los sistemas de transporte a través de costos, usos de energía, emisiones y accesibilidad. Con los indicadores y las diferentes relaciones existentes en los planes utilizados, se visualiza la idea de hacer una modificación a la estructura institucional para dar seguimiento a la regulación y a los resultados de la movilidad urbana.

El segundo caso de Latinoamérica es la Región Metropolitana de Río de Janeiro, Brasil, el cual retoma los cuatro indicadores para medir los impactos del sistema de transporte utilizados en Bogotá; es relevante debido a que existe una propuesta de lineamientos para la coordinación e intervención de los tres niveles de gobierno y de las 
instituciones correspondientes en un Plan Director del Transporte Urbano de la Región Metropolitana de Río de Janeiro; uno de los más importantes y complejos para las acciones de las metrópolis. Con este caso de estudio, el autor contribuye a su metodología de análisis la construcción de escenarios que permitan aportar alternativas para formular los lineamientos y directrices del plan de movilidad urbana de la ZMC.

Concerniente al ámbito nacional, Calderón nos expone como primer caso la ciudad de León en el estado de Guanajuato; se aborda el Plan Maestro de Transporte y el Estudio de Diseño Funcional Detallado del Sistema Integrado de Transporte en el que se plantea un sistema de transporte tronco alimentador que utiliza unidades de alta capacidad (articulados); con ello, retoma la propuesta de lineamientos en relación con el diseño de proyecto, la teoría y las acciones. El segundo caso nacional es la ciudad de Guadalajara en el estado de Jalisco; se rescatan dos indicadores adicionales: el parque vehicular y la accidentabilidad; del plan de movilidad urbana también se utiliza la propuesta de tres proyectos integrales para los diferentes modos de transporte: tren suburbano, macro libramiento y corredores de movilidad, por lo que esta información se considera como referente para estructurar la propuesta de lineamientos.

El capítulo dos plantea los casos de éxito de planes de movilidad urbana de forma clara y concreta para que el lector pueda ubicarse rápidamente en el contexto de la investigación; asimismo, de manera dinámica, retoma las características principales de cada plan y ciudad para aclarar lo que de ellos destaca el autor y así considerarlos en su metodología; los ámbitos que se toman son importantes a nivel internacional, sobre todo los casos de Latinoamérica, pues se acercan un poco más a la realidad de las zonas metropolitanas de México.

El capítulo tres, Diagnóstico de la movilidad urbana en la ZMC, destaca las características del entorno urbano relacionado con la movilidad urbana; expone temas en torno a la configuración de la ZMC, el funcionamiento de vialidades, el cruce de peatones y ciclovías, los motivos de los viajes y estudio de origen y destino de los habitantes de la Zona Metropolitana, así como el transporte público y aforo vehicular. El autor destaca los conflictos existentes en las vialidades, el transporte público y la planeación urbana y demuestra que existe un deficiente sistema vial, así como un sistema urbano ineficiente. Si bien en este capítulo presenta la metodología empleada, el investigador que desee replicar su trabajo metodológico, quizá encuentre parte de la información de manera dispersa en relación con el capítulo dos, donde plantea la utilización de indicadores que pareciera utilizará en el diagnóstico, sin embargo, estos no son retomados puntualmente.

El capítulo cuatro, intitulado Propuesta de lineamientos básicos para la integración de un plan de movilidad urbana (PMU) en la ZMC, concentra los resultados de la investigación en una propuesta de lineamientos que permitan construir los aspectos básicos 
para el desarrollo del plan de movilidad urbana en la ZMC; además, es relevante el marco normativo de los planes de movilidad urbana a niveles federal y estatal, pero se mencionan de manera general.

Resultan de interés los esquemas que el autor considera y retoma de los Planes Integrales de Movilidad. Lineamientos para una movilidad urbana sustentable de la Embajada británica en México y Centro EURE, México, 2012, los cuales soportan el plan de trabajo para la elaboración del plan de movilidad urbana de la ZMC, en el que considera la participación ciudadana. Para elaborar el plan, utiliza cuatro lineamientos: 1) principios orientados de un plan de movilidad urbana, 2) diagnóstico, 3) elaboración del plan de movilidad urbana, y, 4) monitoreo y evaluación.

El libro presenta las conclusiones generales que el autor obtiene de la investigación; culmina de manera paralela a lo abordado en los cuatro capítulos del libro haciendo referencia a la importancia de la teoría del desarrollo urbano, la movilidad urbana y las zonas metropolitanas; destaca la importancia del conocimiento y del manejo teórico de los conceptos y elementos que configuran una metrópoli, ya que esto permite comprender los fenómenos y procesos urbanos, así como de movilidad.

Al entender el fenómeno urbano se pueden explicar los planes de desarrollo urbano y movilidad urbana existentes como instrumentos de planeación urbana. Con la revisión de los casos de estudio y del diagnóstico de la ZMC, Calderón demuestra y concluye que el modelo urbano sobre movilidad urbana actual de la ZMC trae consecuencias negativas, principalmente para la equidad social, el acceso desigual al espacio social y al tiempo; todo esto aunado a que la infraestructura se encuentra envejecida y no logra cubrir las necesidades de movilidad urbana de su población creciente; además, el sistema de transporte es insuficiente y no garantiza un modelo integral que considere orden, seguridad y eficiencia.

A manera de comentarios generales, el libro cuenta con un título propositivo que desde su lectura y portada atrapa al lector para encontrar la receta mágica en la aplicación de la metodología, a fin de desarrollar e implementar un plan de movilidad urbana. Al revisar la estructura y el contenido del índice, la expectativa crece y de inmediato realizamos una lectura de exploración que da buen sabor de boca para después retomar con calma los apartados que el autor refiere.

El capítulo uno presenta un buen panorama; existe una amplia bibliografía en materia de urbanismo, movilidad urbana, desarrollo urbano, transporte y metropolización, sobre todo en temas específicos que se abordan en el capítulo tres en relación con los estudios de origen y destino, así como de infraestructura y equipamiento urbano; uno de los más importantes, quizá de manera general, es Molinero (2005). 
Por otro lado, en búsqueda de un hilo conductor entre los capítulos uno, referido al aspecto teórico; el dos, que aborda los casos de estudio; el tres, que presenta el diagnóstico; y el cuatro, en donde se realiza la propuesta del plan de movilidad urbana, sería bueno contar con una discusión e, incluso, una matriz de análisis conceptual entre los elementos de importancia que exponen los autores retomados con lo que se presenta en los casos de estudio, aclarar si existen coincidencia o no, en el diagnóstico y cómo todo ello contribuye en la propuesta.

Con lo antes expuesto, las virtudes de la obra dan motivos suficientes para que el lector, sea especializado o no, se interese en el tema de la movilidad urbana en las zonas metropolitanas y con ello contribuya a la discusión intelectual de los tópicos relacionados; por lo tanto, este libro es recomendable para bibliografía a nivel licenciatura y posgrado, así como de gran apoyo a los servidores públicos vinculados con la temática, pues encontrarán aspectos teóricos y bibliografía que ampliará sus estudios, la exploración de nuevas líneas de investigación y la aplicación de lineamientos básicos respecto a la integración de un plan de movilidad urbana. 